\title{
Short-time effect of a single session of intense whole-body electromyostimulation on energy expenditure. A contribution to fat reduction?
}

\begin{tabular}{|c|c|}
\hline Journal: & Applied Physiology, Nutrition, and Metabolism \\
\hline Manuscript ID & apnm-2017-0602.R1 \\
\hline Manuscript Type: & Brief communication \\
\hline Date Submitted by the Author: & $15-N o v-2017$ \\
\hline Complete List of Authors: & $\begin{array}{l}\text { Teschler, Marc; Friedrich-Alexander-Universitat Erlangen-Nurnberg Institut } \\
\text { fur Medizinische Physik } \\
\text { Wassermann, Alfred; Insitute of Mathematics, University Bayreuth } \\
\text { Weissenfels, Anja; Friedrich-Alexander-Universitat Erlangen-Nurnberg } \\
\text { Institut fur Medizinische Physik } \\
\text { Fröhlich, Michael; Technische Universitat Kaiserslautern, Department of } \\
\text { Sports Science } \\
\text { Kohl, Matthias; Hochschule Furtwangen, Faculty of Medical and Life Science } \\
\text { Bebenek, Michael; Friedrich-Alexander-Universitat Erlangen-Nurnberg } \\
\text { Institut fur Medizinische Physik } \\
\text { von Stengel, Simon; Friedrich-Alexander-Universitat Erlangen-Nurnberg } \\
\text { Institut fur Medizinische Physik } \\
\text { Kemmler, Wolfgang; Friedrich-Alexander-Universitat Erlangen-Nurnberg } \\
\text { Institut fur Medizinische Physik }\end{array}$ \\
\hline Keyword: & $\begin{array}{l}\text { whole-body electromystimulation, WB-EMS, total energy expenditure, } \\
\text { resting metabolic rate, body composition }\end{array}$ \\
\hline $\begin{array}{r}\text { Is the invited manuscript for } \\
\text { consideration in a Special } \\
\text { Issue? : }\end{array}$ & N/A \\
\hline
\end{tabular}


1 Short-time effect of a single session of intense whole-body 2 electromyostimulation on energy expenditure. A contribution to fat 3 reduction?

4

5 Marc Teschler ${ }^{1}$, Alfred Wassermann ${ }^{2}$, Anja Weissenfels ${ }^{1}$, Michael Fröhlich $^{3}$, Matthias Kohl ${ }^{4}$, Michael

6 Bebenek $^{1}$, Simon von Stengel ${ }^{1}$ and Wolfgang Kemmler ${ }^{1}$

$7 \quad{ }^{1}$ Institute of Medical Physics, Friedrich-Alexander University Erlangen-Nürnberg, Germany

$8 \quad{ }^{2}$ Institute of Mathematics, University of Bayreuth

$9{ }^{3}$ Department of Sports Science, University of Kaiserslautern, Germany

$10{ }^{4}$ Faculty for Medical and Life Science, University Furtwangen, Germany

Corresponding author:

Marc Teschler, Institute of Medical Physics

Friedrich-Alexander University Erlangen-Nürnberg (FAU)

Henkestrasse 91

91054 Erlangen, Germany

Tel: 09131-8522840

Fax: 09131-8522824

Email: marc.teschler@fau.de

Running Title: Energy expenditure and WB-EMS 


\section{ABSTRACT}

25 To determine the underlying mechanisms after one session of (intense) whole-body 26 electromyostimulation (WB-EMS) on total energy expenditure (TEE) and resting metabolic rate 27 (RMR), 16 subjects followed a standardized protocol of indirect calorimetry for up to $72 \mathrm{~h}$ in $12 \mathrm{~h}$ 28 intervals. The single session significantly increased RMR about $25 \pm 10 \%(p<0.001)$ and TEE for about $299.5 \pm 1 \%$, a net effect of $\sim 460 \pm 50 \mathrm{kcal}$ (WB-EMS vs. CG).

\section{KEY WORDS}

31 whole-body electromyostimulation; WB-EMS; total energy expenditure; resting metabolic rate; RMR; fat reduction; body composition 
INTRODUCTION

Whole-body electromyostimulation (WB-EMS) might be an alternative to conventional resistance training (RT) for improving body composition. With respect to body-fat parameters, a similar 14week randomized controlled trial reported similar significant and clinically relevant reductions after high-intensity RT (HIT) and WB-EMS (Kemmler et al. 2016a) that even (slightly) exceed the increases of muscle mass. Addressing acute exercise effects, a single session of 20 min WB-EMS increases EE by up to $470 \mathrm{kcal} / \mathrm{h}$ (Boccia et al. 2016; Kemmler et al. 2012), thus WB-EMS can be considered as a highintensity training setting (Haskell et al. 2007). Further, WB-EMS significantly affects Resting Metabolic Rate (RMR) through its significant impact on muscle mass (Kemmler et al. 2016a). However, neither the acute nor chronic mechanisms (via RMR) of WB-EMS-induced increases of EE (Kemmler et al. 2010; Kemmler et al. 2012) can fully explain the significant and clinically relevant body-fat reduction during WB-EMS applications, so it is important to further determine the "missing link", which might be the immediate post-exercise effects of WB-EMS on EE. Therefore, it was the aim of this study to determine the short-/medium-term effects of one single session of an intense but otherwise conventional WB-EMS application on EE.

Our hypotheses are (1) that a single session of WB-EMS induces significant short-term effects on EE and (2) that these significant effects on EE last up to 60 hours post-exercise.

\section{MATERIALS and METHODS}

The project was realized in a randomized parallel group, crossover design with each participant $(n=$ 16) undergoing all the RMR assessments (pre, 1, 12, 24, 36, 48, 60, 72h post) twice, thus acting as their own control. The project was conducted between February and June 2016 by the Institute of Medical Physics (IMP), University of Erlangen-Nürnberg (FAU) Germany. The study complied with the Helsinki Declaration "Ethical Principles for Medical Research Involving Human Subjects" and was approved by the ethical committee of the FAU (No. 183_14B). All participants gave their written informed consent. 
Primary study outcome:

- Total energy expenditure (TEE) with and without previous WB-EMS application determined via indirect calorimetry.

\section{Secondary study outcomes:}

- Changes of RMR and EE with and without previous WB-EMS application determined via indirect calorimetry.

There were no changes in the outcomes after trail commencement.

\section{Participants}

16 eligible participants (13 men, 3 women; age: $33.5 \pm 9.6$ years, weight: $79.5 \pm 12.4 \mathrm{~kg}$, height: $180.2 \pm 8.2 \mathrm{~cm}$, SMM: $37.4 \pm 6.4 \mathrm{~kg}$, training volume: $327 \pm 128 \mathrm{~min} /$ week) were included if they (a) were healthy, 20-50 years old, (b) frequently conducted RT ( $\geq 2$ sessions/week) and (c) were experienced in WB-EMS. Subjects were excluded under the following criteria: (a) medication/diseases affecting muscle metabolism or renal function, or (b) conditions that prevent WB-EMS (e.g. cardia arrhythmia) and (c) inability to attend all the RMR assessments within a variance of $\pm 1 h$.

\section{Assessment}

Height was determined with a stadiometer (Holtain Ltd. Crymmych, Wales), weight, muscle mass and body fat were assessed via multi-frequent bioelectrical impedance analysis (DSM-BIA, InBody770, BioSpace, Seoul, Korea).

Indirect calorimetry was used to determine RMR and EE (Oxycon Mobil, Viasys, Conshohocken, PA, U.S.). $\mathrm{VO}_{2}, \mathrm{VCO}_{2}, \mathrm{VE}$ were continuously determined breath by breath. The $\mathrm{HR}$ was continuously evaluated using a Polar tester (Polar RS 800, Kempele, Finland). Test were performed pre- and immediately post-intervention and further in $12 \mathrm{~h}$ cycles for $72 \mathrm{~h}$ (supplementary figure S1). After resting for $10 \mathrm{~min}$ in a supine position in a semi-dark room $\left(23^{\circ} \mathrm{C}\right)$, data of the subsequent 20 min was 
82 included in the analysis. Participants were asked to rest and do not move or talk during the test.

Participants were required to avoid intense physical activity, drugs and unusual nutrition $24 \mathrm{~h}$ prior to the tests. Equipment was calibrated according to the manufacturer's recommendations before each test.

\section{WB-EMS application}

The WB-EMS application was conducted with a miha bodytec ${ }^{\circledR}$ Type I device (Gersthofen, Germany) consisting of a control panel and a set of electrodes. The system allows simultaneous stimulation of up to 10 large muscle groups (Kemmler and von Stengel 2013) with regionally dedicated current intensity.

We applied a bipolar WB-EMS protocol (20min, $85 \mathrm{~Hz}, 350 \mu \mathrm{s}$, 1s ramp, 6s impulse - 4s break) (Filipovic et al. 2012) that stimulates 8 large muscle groups. During the impulse phase participants performed (very) slight movement patterns (e.g. dynamic squatting with knee ankle: $\geq 120^{\circ}$ ), standardized by a synchronized video. Participants completed 1-2x 6-8 repetitions of 8 movements. Certified instructors encouraged the participants to work with (very) high effort. Intensity was increased every 3-5min to maintain the prescribed perceived exertion of "7"-" 8 " (very hard-very hard+) on the Borg CR-10 scale (Borg and Kaijser 2006). Of importance, we did not apply the same movements (without WB-EMS) during the control conditions.

\section{Statistics}

In order to prove our hypothesis, quantified as a $10 \%$ higher TEE in the WB-EMS compared with the CG, 14 subjects had to be included to generate a power of $95 \%$ ( $\alpha=.05$ ) (Jamurtas et al. 2004).

The EE curves were approximated by linear interpolation of the six measurements taken at $12 \mathrm{~h}$ intervals. Then, the areas under curve (AUC) were determined by integration of the resulting piecewise linear curves, i.e. $A U C=12 *\left(1 / 2 * E E \_p o s t+E E \_12+E E \_24+E E \_36+E E \_48+E E \_60+\right.$ 1/2*EE_72). 
Data are consistently reported as mean and standard deviations (MV $\pm \mathrm{SD})$. Depending on the

107 distribution of the data, we used student's t-tests or Wilcoxon rank-tests to analyze changes within

108 groups. The differences between both conditions were evaluated using the Welch t-test. Significance

109 was accepted at $p<.05$. Effect size (ES) was calculated by using Cohen d' and SPSS 22 (IBM, Chicago,

110 USA) was used for all statistical procedure.

\section{RESULTS}

112 Table 1 listed the results for the total cumulated EE (TEE in total kcal) during the $72 \mathrm{~h}$ assessment

113 phase determined by the AUC. In summary, the difference for cumulated TEE between WB-EMS and

114 control condition averaged $460 \pm 51 \mathrm{kcal}$. Thus, hypothesis 1 that EMS induces significant short-term

115 effects on EE can be confirmed.

116 Supplementary figure S2 shows the percentage changes of RMR (from baseline) for all time intervals

117 during EMS and control condition along with the corresponding group differences. RMR after WB-

118 EMS showed a highly significant $25 \%$ increase from pre- to immediately post-exercise $(82.7 \pm 6.9$ to

$119103.5 \pm 11.4 \mathrm{kcal} / \mathrm{h} ; \mathrm{p}<.001$ ). RMR subsequently decreased almost linearly (supplementary figure S2),

120 however we observed statistically significant group differences for all periods up to $48 \mathrm{~h}$ : $1 \mathrm{~h}$ :

$121103.5 \pm 11.4$ vs. $88.7 \pm 8.9 \mathrm{kcal} / \mathrm{h}(\mathrm{p}<.001) ; 12 \mathrm{~h}: 100.1 \pm 12.7$ vs. $86.9 \pm 10.3 \mathrm{kcal} / \mathrm{h}(\mathrm{p}<.001) ; 24 \mathrm{~h}$ :

$12297.3 \pm 11.7$ vs. $87.1 \pm 10.4 \mathrm{kcal} / \mathrm{h}(<.001) ; 36 \mathrm{~h}: 93.7 \pm 11.0$ vs. $88.6 \pm 9.9 \mathrm{kcal} / \mathrm{h}(\mathrm{p}<.001) ; 48 \mathrm{~h}: 90.5 \pm 10.8$

123 vs. $89.1 \pm 10.1 \mathrm{kcal} / \mathrm{h}(\mathrm{p}=.014)$. Nevertheless, differences after $60 \mathrm{~h}$ were borderline non-significant

$124 \quad(p=.052)$, so we have to reject hypothesis 2 .

125 Of importance for the interpretation of the results, the respiratory exchange ratio (RER) increased

126 significantly from pre- to post intervention $(.89 \pm .05$ to $.94 \pm .06, p=.001)$, and further decreased

127 significantly for $48 \mathrm{~h}-60 \mathrm{~h}$ with significant lower levels compared to pre-exercise $(.86 \pm .05, \mathrm{p}=.034$;

$128.87 \pm .04, p=.078)$. No corresponding variations were determined for the control condition. 
129

130

131

132

133

134

\section{DISCUSSION}

WB-EMS is a promising exercise technology that favorably affects body composition (Kemmler et al. 2010; Kemmler et al. 2016a; Kemmler et al. 2016b; Kemmler and von Stengel 2013). However, the time efficiency of WB-EMS with $20 \mathrm{~min} /$ session applied 1.5 times/week, hardly correlates with the significant fat reduction in cohorts of normal-weight, over-weight and obese people (Kemmler et al. 2016a; Kemmler and von Stengel 2013; Kemmler et al. 2017). Thus, it was the aim of this study to close the gap between acute and long-term EE effects after WB-EMS evaluated in earlier studies (Kemmler et al. 2010; Kemmler et al. 2012) .

With respect of the short-term effect of WB-EMS, there is considerable evidence that intense WBEMS needs prolonged periods of resynthesis, repair and adaptation (Teschler et al. 2016). Indeed, in the present study the EE value peaked $25 \%$ above baseline immediately post WB-EMS $(p<.001)$ and remained significantly increased for up to $48 \mathrm{~h}$ post exercise. In total, during the observational period of $72 \mathrm{~h}$ the effect on EE (WB-EMS vs. control) averaged around $460 \mathrm{kcal}$. We further observed a significantly enhanced post-exercise lipid oxidation that remained for 48-60h, a result that confirmed data observed after conventional RT (e.g: Gillette et al. 1994; Melby et al. 1993; Paschalis et al. 2010).

Due to divergent interventions and EPOC observation length (60min (Haltom et al. 1999) up to $72 \mathrm{~h}$ (Hackney et al. 2008)) it is difficult to compare our result with most of the recent studies. However, some studies applied intense RT with post-exercise observation periods comparable to this contribution.

After high-volume RT at 70\% 1-repetition-maximum (1RM), significant increases in RMR ( $\approx 5 \%)$ up to 15h (Gillette et al. 1994; Melby et al. 1993 ) were reported. In addition, ten upper and lower body exercises induced significant $\mathrm{RMR}$ elevation after $10 \mathrm{~h}(\approx 7.6 \%)$ that lasted up to $24 \mathrm{~h}(\approx 5.5 \%)$ (Jamurtas et al. 2004), whereas powerlifting training (four circuits of bench press, power cleans and 
squatting) with $\approx 8-12$ repetitions $(70-80 \% 1 \mathrm{RM}$; 2 -min rest period) reported an increased $\mathrm{RMR}$

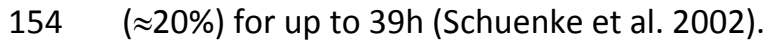

155

156

157

158

159

160

161

162

163

164

165

166

167

168

169

170

171

172

173

174

175

176

177

RT with emphasis on the eccentric phase (leg-press, 8 sets at 6 RM, 1s concentric/4s eccentric) induced a RMR increase of $8-9 \%$ in trained and untrained subjects $48 \mathrm{~h}$ post exercise (Dolezal et al. 2000). However, the trial most similar to our result applied a high volume, whole-body RT (4 upper and 4 lower exercises, 8 sets, 6 repetitions with 1s concentric/3s eccentric) and reported a significant 9.2\% EE increase in untrained and $7.9 \%$ in trained subjects that remained up to $72 \mathrm{~h}$ (Hackney et al. 2008). Also comparable to our results, isokinetic leg press exercises with emphasis on the eccentric phase ( 5 sets, 15 repetitions with MVC, 2 min break) significantly raised RMR for up to $48 \mathrm{~h}(\approx 9 \%)$ in lean and for up to $72 \mathrm{~h}$ in obese subjects ( $\approx 25 \%)$ (Paschalis et al. 2010).

Thus, the scientific evidence so far suggests that strenuous RT-related exercise elevates post-exercise EE for a prolonged period. Additionally, the enhanced fat oxidation observed in this study and confirmed by RT-studies (Gillette et al. 1994; Melby et al. 1993; Paschalis et al. 2010) during the postexercise period contributes to explaining the pronounced fat reduction after WB-EMS.

One study limitation may hinder the generality of our result. Subjects included were predominately infrequent WB-EMS applicants (1-2 sessions/month), who may be more responsive to the relatively unusual WB-EMS stimuli. Indeed, an earlier study (Teschler et al. 2016) with WB-EMS novices resulted in a very pronounced repeated bout effect, with a 20 -fold reduction of initial CK peak values after 10-weeks of WB-EMS conditioning. Thus, familiarization might result in reduced WB-EMSinduced elevations of EE.

Not relevant for the present study, but a limitation of our general approach to determining acute, short- and long-term WB-EMS effects on EE might be that we do not consistently involve the same cohort. While the participants during the evaluation of acute and short time effects largely overlapped, long-term effects of WB-EMS on EE were determined with a postmenopausal cohort, potentially with an age-dependent lower potential for exercise induced-adaptation. However, the 
comparison of two WB-EMS trials that applied the same exercise protocol, albeit with younger (Kemmler et al. 2010) or older men (Kemmler et al. 2016a), resulted in comparable changes of body composition (i.e. LBM and body fat).

In summary, the pronounced WB-EMS-induced reduction of body fat can be largely explained by acute, short- and long-term effects of regular (1.5 x 20min sessions/week) WB-EMS application on EE. Thus, a carefully supervised and guided WB-EMS application might be a safe, effective and very time-efficient option not only, but also to reduce body fat predominately for people unable or unwilling to exercise conventionally.

\section{ACKNOWLEDGEMENTS}

The authors declare that there are no conflicts of interest.

The present work was performed in (partial) fulfillment of the requirements for obtaining the degree "Dr. rer. biol. hum".

\section{REFERENCES}

Boccia, G., Fornasiero, A., Savoldelli, A., Bortolan, L., Rainoldi, A., Schena, F., et al. 2016. Oxygen consumption and muscle fatigue induced by whole-body electromyostimulation compared to equal-duration body weight circuit training. Sport Sciences for Health, doi:10.1007/s11332016-0335-4.

Borg, E. and Kaijser, L. 2006. A comparison between three rating scales for perceived exertion and two different work tests. Scand. J. Med. Sci. Sports, 16(1): 57-69.

Dolezal, B. A., Potteiger, J. A., Jacobsen, D. J. and Benedict, S. H. 2000. Muscle damage and resting metabolic rate after acute resistance exercise with an eccentric overload. Med. Sci. Sports Exerc. 32(7): 1202-1207.

Filipovic, A., Kleinoder, H., Dormann, U. and Mester, J. 2012. Electromyostimulation - a systematic review of the effects of different electromyostimulation methods on selected strength parameters in trained and elite athletes. J. Strength Cond. Res. 26(9): 2600-2614.

Gillette, C. A., Bullough, R. C. and Melby, C. L. 1994. Postexercise energy expenditure in response to acute aerobic or resistive exercise. Int. J. Sport Nutr. 4(4): 347-360.

Hackney, K. J., Engels, H. J. and Gretebeck, R. J. 2008. Resting energy expenditure and delayed-onset muscle soreness after full-body resistance training with an eccentric concentration. J. Strength Cond. Res. 22(5): 1602-1609.

Haltom, R. W., Kraemer, R. R., Sloan, R. A., Hebert, E. P., Frank, K. and Tryniecki, J. L. 1999. Circuit weight training and its effects on excess postexercise oxygen consumption. Med. Sci. Sports Exerc. 31(11): 1613-1618. 
Haskell, W. L., Lee, I. M., Pate, R. R., Powell, K. E., Blair, S. N., Franklin, B. A., et al. 2007. Physical activity and public health: updated recommendation for adults from the American College of Sports Medicine and the American Heart Association. Med. Sci. Sports Exerc. 39(8): 14231434.

Jamurtas, A. Z., Koutedakis, Y., Paschalis, V., Tofas, T., Yfanti, C., Tsiokanos, A., et al. 2004. The effects of a single bout of exercise on resting energy expenditure and respiratory exchange ratio. Eur. J. Appl. Physiol. 92(4-5): 393-398.

Kemmler, W., Schliffka, R., Mayhew, J. L. and von Stengel, S. 2010. Effects of whole-body electromyostimulation on resting metabolic rate, body composition, and maximum strength in postmenopausal women: the Training and ElectroStimulation Trial. J. Strength Cond. Res. 24(7): 1880-1887.

Kemmler, W., Teschler, M., Weissenfels, A., Bebenek, M., Fröhlich, M., Kohl, M., et al. 2016a. Effects of Whole-Body Electromyostimulation versus High-Intensity Resistance Exercise on Body Composition and Strength: A Randomized Controlled Study. Evid. Based Complement. Alternat. Med. 2016: 9236809. PMID:4789460.

Kemmler, W., Teschler, M., Weissenfels, A., Bebenek, M., von Stengel, S., Kohl, M., et al. 2016b. Whole-body electromyostimulation to fight sarcopenic obesity in community-dwelling older women at risk. Resultsof the randomized controlled FORMOsA-sarcopenic obesity study. Osteoporos. Int. 27(11): 3261-3270.

Kemmler, W. and von Stengel, S. 2013. Whole-body electromyostimulation as a means to impact muscle mass and abdominal body fat in lean, sedentary, older female adults: subanalysis of the TEST-III trial. Clin. Interv. Aging, 8: 1353-1364. PMID:3795534.

Kemmler, W., von Stengel, S., Schwarz, J. and Mayhew, J. L. 2012. Effect of whole-body electromyostimulation on energy expenditure during exercise. J. Strength Cond. Res. 26(1): 240-245.

Kemmler, W., Weissenfels, A., Teschler, M., Willert, S., Bebenek, M., Shojaa, M., et al. 2017. Wholebody Electromyostimulation and protein supplementation favorably affect Sarcopenic Obesity in community-dwelling older men at risk. The Randomized Controlled FranSO Study. Clin. Interv. Aging, 12: 1503-1513, doi:10.2147/CIA.S137987. PMID:5624743

Melby, C. L., Scholl, C., Edwards, G. and Bullough, R. 1993. Effect of acute resistance exercise on postexercise energy expenditure and resting metabolic rate. J. Appl. Physiol. 75: 1847-1853.

Paschalis, V., Nikolaidis, M. G., Giakas, G., Theodorou, A. A., Sakellariou, G. K., Fatouros, I. G., et al. 2010. Beneficial changes in energy expenditure and lipid profile after eccentric exercise in overweight and lean women. Scand. J. Med. Sci. Sports, 20(1): e103-111.

Schuenke, M. D., Mikat, R. P. and McBride, J. M. 2002. Effect of an acute period of resistance exercise on excess post-exercise oxygen consumption: implications for body mass management. Eur. J. Appl. Physiol. 86(5): 411-417.

Teschler, M., Weissenfels, A., Fröhlich, M., Kohl, M., Bebenek, M., von Stengel, S., et al. 2016. (Very) High creatine kinase (CK) levels after Whole-Body Electromyostimulation. Are there implications for health? Int. J. Clin. Exp. Med. 9(11): 22841-22850. 
Tables

Table 1. Mean values (MV) and standard deviation (SD) of the cumulated TEE (kcal) structured for 12h-interval; significance (p)

\begin{tabular}{|c|c|c|c|}
\hline & $\begin{array}{c}\text { WB-EMS (n=16) } \\
\text { (MV } \pm \text { SD) }\end{array}$ & Non-WB-EMS (n=16) & $p$ \\
& $(M V \pm S D)$ & \\
\hline
\end{tabular}

Cumulated total energy expenditure [kcal]

\begin{tabular}{|l|l|l|l|}
\hline $\mathbf{1 2} \mathbf{h}$ & $1,218 \pm 147$ & $1,052 \pm 108$ & .001 \\
\hline $\mathbf{2 4} \mathbf{h}$ & $2,402 \pm 276$ & $2,097 \pm 222$ & .002 \\
\hline $\mathbf{3 6} \mathbf{h}$ & $3,548 \pm 389$ & $3,150 \pm 334$ & .004 \\
\hline $\mathbf{4 8} \mathbf{h}$ & $4,666 \pm 486$ & $4,197 \pm 430$ & .007 \\
\hline $\mathbf{6 0} \mathbf{h}$ & $5,744 \pm 572$ & $5,244 \pm 526$ & .015 \\
\hline $\mathbf{7 2} \mathbf{h}$ & $6,778 \pm 671$ & $6,315 \pm 610$ & .050 \\
\hline
\end{tabular}

